\title{
A Frequency Domain Equalizer for WLAN 802.11g Single-Carrier Transmission Mode
}

\author{
Frank H. Hsiao \\ Department of Computer Science and Information \\ National Chiao Tung University \\ Hsinchu, Taiwan (ROC) \\ frankie_917@hotmail.com
}

\author{
Terng-Yin Hsu \\ Department of Computer Science and Information \\ National Chiao Tung University \\ Hsinchu, Taiwan (ROC) \\ tyhsu@si2lab.org
}

\begin{abstract}
In this paper a frequency domain equalizer is proposed for WLAN 802.11g single-carrier transmission mode. Channel estimation is achieved with modified recursive least square (RLS) algorithm in frequency domain. For channel compensation, OFDM like frequency domain zero-forcing equalization is performed with FFT units and complex divider. In 802.11g single-carrier mode, transmission packets are not equipped with cyclic prefixes so signal overlapping and discarding using sliding windows are maintained at each equalization process to decrease the symbol estimation error caused by circularly de-convoluting a linear channel convolution process. With the equalizer of single-carrier mode implemented in frequency domain many of their computation resources can be shared with 802.11g OFDM equalizer offering a low complexity multimode receiver solution.
\end{abstract}

\section{INTRODUCTION}

IEEE $802.11 \mathrm{~g}$ standard in $2.4 \mathrm{GHz}$ band has been established to accommodate high bit rate demanding applications supporting OFDM technology used by WLAN 802.11a systems and offering backward compatibility with the legacy $802.11 \mathrm{~b}$ single-carrier systems [1]. WLAN $802.11 \mathrm{~g}$ can offer rates from $1 \mathrm{Mbps}$ up to $54 \mathrm{Mbps}$ and capable of operating in single-carrier and multi-carrier modes such as ERP(Extended Rate PHY)-DSSS/CCK, ERPOFDM, ERP-PBCC and CCK-OFDM handling BPSK, DBPSK, QPSK, DQPSK, 8-PSK, 16-QAM, and 64-QAM modulation schemes. DSSS/CCK transmission mode which is a mandatory part of single-carrier $802.11 \mathrm{~b}$ systems has traditionally implemented equalizers in time domain with RAKE receiver or DFE [2][3]. However, for a 802.11g multimode receiver straight forward implementation of time domain equalizer for the single-carrier DSSS/CCK mode and frequency domain implementation of OFDM equalizer may not be economic considering overall hardware complexity. By implementing the equalizer of $802.11 \mathrm{~g}$ single-carrier mode in frequency domain making the full use of the FFT units provided in OFDM mode, the overall complexity of the multimode receiver can be reduced through hardware sharing
In this paper channel estimation for this single-carrier equalizer is proposed based on modified recursive least square (RLS) algorithm [4]. The channel estimation filter operates in frequency domain and it adaptively models the channel impulse response under noise. Although RLS adaptive algorithms compared to LMS ones are higher in computation cost they can achieve convergence at a faster rate giving more design flexibility for short preamble $\mathrm{PBCC}$ transmission mode [1]. Following channel estimation, issues of channel equalization are discussed. In DSSS/CCK singlecarrier mode the transmission packet does not possess any cyclic prefixes so direct frequency domain zero-forcing equalization will result in symbol estimation error since linear convoluted data may not be properly de-convoluted in frequency domain without adding any transmission redundancy. Signal overlapping and discarding using sliding windows are maintained at each equalization process to minimize the symbol estimation error.

This paper is organized as follows: Single-carrier DSSS/CCK transmission parameters and channel estimation are described in section II. Section III discusses zero-forcing equalization in frequency domain. Simulation results and discussions are presented in section IV and finally conclusions in section $\mathrm{V}$.

\section{FREQUENCY DOMAIN CHANNEL ESTIMATION}

\section{A. $\quad 802.11 \mathrm{~g}$ Single-carrier System Model}

In $802.11 \mathrm{~g}$ single-carrier $\mathrm{DSSS} / \mathrm{CCK}$ mode the transmission packet consists of a preamble sequence of 144 bits used for synchronization, channel estimation and other front end signal processing, followed by a 48 bit header used for packet detection and finally payload of variable sizes. Preamble and header are always transmitted at 1 Mbps and they are always encoded by DBPSK and spread out with 11 chip Barker sequence $b=\{1,-1,1,1,-1,1,1,1,-1$,$1,-1\}$ using DSSS technique at a chip rate of $11 \mathrm{Mbps}$. The payload symbols can be encoded with various modulation to 
meet the application requirement. Fig. 1 below shows the transmitted packet format.

\begin{tabular}{|c|c|c|c|c|c|}
\hline $\begin{array}{c}\text { SYNC } \\
128 \text { bits }\end{array}$ & $\begin{array}{c}\text { SFD } \\
16 \text { bits }\end{array}$ & $\begin{array}{c}\text { SIGNAL } \\
8 \text { bits }\end{array}$ & $\begin{array}{c}\text { SERVICE } \\
8 \text { bits }\end{array}$ & $\begin{array}{c}\text { LENGTH } \\
16 \text { bits }\end{array}$ & $\begin{array}{c}\text { CRC } \\
16 \text { bits }\end{array}$ \\
\hline \multicolumn{4}{|c|}{$\begin{array}{c}\text { Header } \\
48 \text { bits }\end{array}$} & Payload \\
\hline
\end{tabular}

Figure 1. IEEE 802.11g ERP DSSS/CCK packet format

\section{B. Channel Estimation}

The proposed frequency domain channel modeling filter based on RLS error criterion has the advantages of using the FFT units already provided in $802.11 \mathrm{~g}$ OFDM mode to perform fast convolution and offering fast convergence. This filter is similar to unconstrained FLMS filter but with the filter coefficients updated using modified RLS algorithm [5]. Fig 2 below shows the overview of the modeling filter used for channel estimation.



Figure 2. Channel modeling filter

The unknown multipath channel, excited by input signal $\boldsymbol{x}(n)$ which are the spread chips of known preamble, is expressed by an FIR filter $\boldsymbol{h}=\left[h_{1}, h_{2}, h_{3}, \ldots, h_{L}\right]$ of length $L$. The output signal $\boldsymbol{y}^{\prime}(n)$ with additive noise $\boldsymbol{v}(n)$ added gives the desired signals $\boldsymbol{d}(n)=\boldsymbol{y}^{\prime}(n)+\boldsymbol{v}(n)$ which are the signals received at the receiver side. The model impulse response of length $L$ is defined as $\boldsymbol{w}=\left[w_{1}, w_{2}, \ldots w_{L}\right]$. In the following we assume a block size equal to length $L$. For fast convolution according to overlap-save method, first transform two concatenated blocks of input signals $\boldsymbol{x}(n)$ to frequency domain,

$$
\boldsymbol{X}(k)=\boldsymbol{F}\{[x(k L-L+1) \ldots x(k L+L)]\}
$$

where $\boldsymbol{F}\{$. $\}$ denotes a $2 L$-point DFT and $k$ is the block index overtime. At time $k$ the estimated channel output signals in frequency domain $\boldsymbol{Y}(k)$ are then generated by multiplying the discrete fourier transform of $\boldsymbol{w}$ with $\boldsymbol{X}(k)$ element-byelement which can be written as

$$
\boldsymbol{Y}(k)=\boldsymbol{W}(k) \boldsymbol{X}(k)
$$

where in (2) $\boldsymbol{W}(k)=\boldsymbol{F}\left\{\left[\begin{array}{ll}\boldsymbol{w}(k) & 0_{1 \times L}\end{array}\right]\right\}=\left[\begin{array}{lll}\left.W_{1}(k)\right) & W_{2}(k) \ldots W_{2 L}(k)\end{array}\right]$ and $0_{1 \times L}$ denotes a zero vector of size $L$. According to overlap-save method, only the last $L$ points of the $\boldsymbol{Y}(k)$ is the result of a linear convolution. The residual error vector $\boldsymbol{e}(k)$ in time domain is obtained by subtracting the desired signals $\boldsymbol{d}(n)$ with the estimated channel output signals windowed by $\boldsymbol{B}=\left[0_{1 \times L} 1_{1 \times L}\right]$ to give the error vector:

$$
\boldsymbol{e}(k)=\left[0_{1 \times L} d(k L+1) \ldots d(k L+L)-\boldsymbol{B} \boldsymbol{F}^{-1} \boldsymbol{Y}(k)\right] .
$$

The error vector in (3) can serve as output signals and as feedback for next adaptation step in order to identify and track the channel impulse response. Transforming the error vector to frequency domain $\boldsymbol{E}(k)=\boldsymbol{F} \boldsymbol{e}(k)$, the channel modeling filter tap weights are adapted with modified RLS. The cost function $\boldsymbol{J}_{j}(k)$ according to $j$ th entry of $\boldsymbol{E}(k)$ is defined as

$$
\boldsymbol{J}_{j}(k)=\sum_{j=1}^{k} \lambda^{k-j}\left|\boldsymbol{E}_{j}(i)\right|^{2},
$$

where $\lambda$ is the forgetting factor and $j$ is the frequency domain index. The update equation of the filter coefficients at time $k$ follows as

$$
\boldsymbol{W}(k+1)=\boldsymbol{W}(k)+g(k) \boldsymbol{E}(k),
$$

where the gain vector $g(k)$ in $(5)$ can be obtained as

$$
g(k)=\frac{1}{\lambda+\boldsymbol{Y}(k) \boldsymbol{U}(k)} \boldsymbol{U}(k)
$$

and the intermediate vector $\boldsymbol{U}(k)$ in (6) is

$$
\boldsymbol{U}(k)=\boldsymbol{P}(k) \boldsymbol{Y}^{*}(k),
$$

where (.) $)^{*}$ denotes the conjugate of a vector (.).The inverse correlation vector $\boldsymbol{P}(k)$ of input data and filter tap weights in (7) is initialized as :

$$
\boldsymbol{P}(1)=\delta^{-1} \times 1_{1 \times 2 L},
$$

where $\delta$ is a small number and $\boldsymbol{P}(k)$ updated in each iteration as :

$$
\boldsymbol{P}(k+1)=\frac{1}{\lambda}[\boldsymbol{P}(k)-g(k) \boldsymbol{U}(k)] .
$$

The setting of $\lambda<1$, will give more weight to the recent samples of the error estimates and provide the channel identification unit with tracking capability. At the end of channel modeling process the estimated time domain 
channel impulse response can be obtained from frequency domain as

$$
\boldsymbol{w}=\boldsymbol{B} \boldsymbol{F}^{-1}\left\{\left[W_{1}, W_{2}, \ldots, W_{2 L}\right]\right\},
$$

where $\mathrm{B}^{\prime}=\left[1_{1 \times L} 0_{1 \times L}\right]$.

\section{Channel Equalization}

After the channel estimation, channel equalization is performed to compensate the multipath distortions. The received data at the receiver are described with vector-matrix notation in (11). In (11) we assume that a data packet $\boldsymbol{d}=\left[d_{1}, d_{2}, \ldots, d_{N}\right]$ of $N$ bits is transmitted over a time invariant channel of length $L$ characterized by vector $\boldsymbol{h}=\left[h_{1}, h_{2} \ldots h_{L}\right]$. The received vector $\boldsymbol{s}=\left[s_{l}, s_{2}, \ldots, s_{N+L-I}\right]$ can be computed by multiplying the Toeplitz channel matrix $H \in^{(N+L-1) \times(N)}$ with the data packet $d$ and adding a noise vector $\boldsymbol{v} \in^{(1) \times(N+L-1)}$ :

$$
\boldsymbol{s}=H \boldsymbol{d}+\boldsymbol{v} \text { and } H=\left[\begin{array}{cccc}
h_{1} & 0 & \cdots & 0 \\
h_{2} & h_{1} & \cdots & 0 \\
\vdots & h_{2} & \cdots & 0 \\
h_{L-1} & \vdots & \ddots & \vdots \\
h_{L} & h_{L-1} & \cdots & 0 \\
0 & h_{L} & \cdots & \vdots \\
\vdots & \vdots & \cdots & h_{L-1} \\
0 & 0 & \cdots & h_{L}
\end{array}\right]
$$

Zero-forcing equalization can be done efficiently in frequency domain [6][7][8], and it reduces to an eigenvalue decomposition process of a circular matrix based on the fact that every circular matrix $\boldsymbol{C}$ can be decomposed by DFT matrices $\boldsymbol{F}$ as:

$$
\boldsymbol{C}=\boldsymbol{F}^{-1} \boldsymbol{C}_{\boldsymbol{F}} \boldsymbol{F},
$$

where $\boldsymbol{C}_{\boldsymbol{F}}=\operatorname{diag}\left(\boldsymbol{F}_{c_{1}}\right), c_{1}$ is the first column of $\boldsymbol{C}$. Substituting (12) into receiving models of the form $H \boldsymbol{x}=\boldsymbol{b}, \mathrm{x}$ can be solved as:

$$
\boldsymbol{x}=\boldsymbol{C}^{-1} \boldsymbol{b}=\boldsymbol{F}^{-1} \boldsymbol{C}_{F}^{-1} \boldsymbol{F} \boldsymbol{b} .
$$

Transmission packets with cyclic prefix or other transmission redundancy making the non-circular channel convolution process appear circular can thus benefit the simple frequency domain zero-forcing equalization [7] [8].

However the transmission packet of single carrier transmission modes in WLAN 802.11g standard does not possess any cyclic prefix or other kinds of transmission redundancy so direct division of the received data and estimated channel impulse response in frequency domain will result in symbol estimation error [8]. This symbol estimation error will remain even with a well estimated channel under high SNR. Fig.3 below shows under multipath and noise the relative distance between the transmitted symbols and symbols after 64-point FFT frequency domain equalization but before demodulation. From Fig.3 it can be seen that without any cyclic prefix or redundancy the estimation errors very with index and the maximum distance occur at the beginning and at end of each block however the relative distances in the middle of each block are much lower.

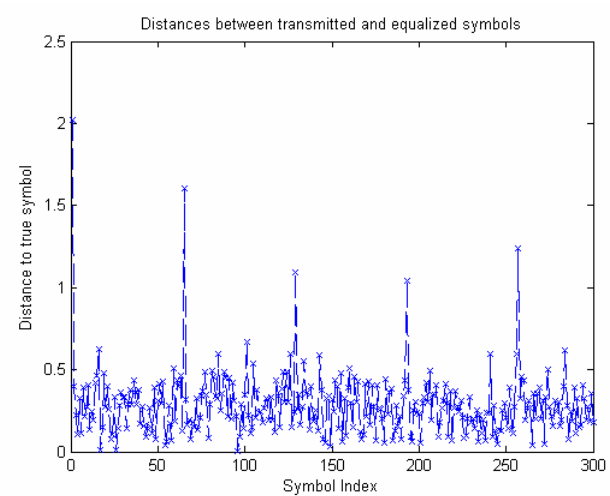

Figure 3. Distance between transmitted and equalized symbols

Since most of the circular effects of the frequency domain division process occur at the edges, by overlapping and sliding-window, the data $d$ can be estimated by solving smaller overlapping sliding windows at a time. Only a partial portion of the data is estimated at each equalization process thus the estimates in the boundary of each window are discarded and estimates at the middle of the window are kept for demodulation. With this scheme the estimation error produced by division process in frequency domain can be decreased. Fig. 4 below shows this scheme.

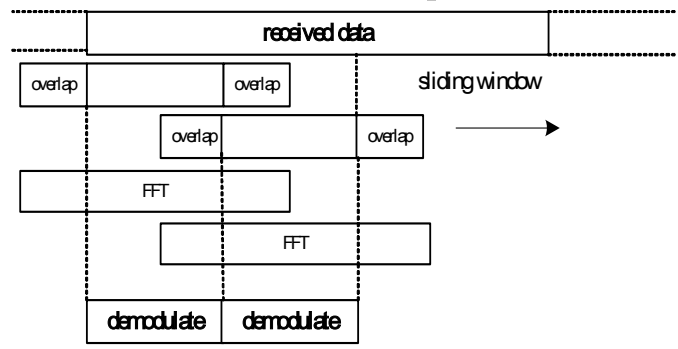

Figure 4. Overlapping sliding window

\section{Simulation Results AND Disscussion}

This section presents some simulation results of the proposed frequency domain equalizer with parameters taken from single carrier 802.11g ERP DSSS/CCK transmission mode. The BER versus SNR curves with perfect channel knowledge and with channel estimation have been evaluated using a channel model suitable for indoor WLAN environment suggested by [9]. The multiapth channel used in simulation is a 8 tap exponential decay channel model with a RMS delay of $100 \mathrm{~ns}$ and $250 \mathrm{~ns}$. A RMS delay of $100 \mathrm{~ns}$ is a 
moderate delay spread environment characterizing an indoor office or conference room and RMS delay of 250ns characterizes difficult indoor environments like a shopping mall or a factory. Fig.4 below shows the non-zero power paths of the multipath channel and the delay between each path is 90.9 ns.

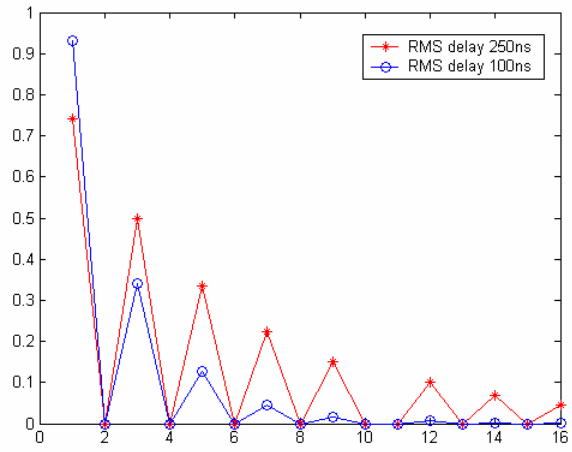

Figure 5. Channel model for RMS delay of 50ns and 100ns

In channel estimation, the length of channel modeling filter is set to 8 taps. At channel equalization the FFT size at each equalization process is set to 64 corresponding with the defined 64-point FFT computation in 802.11g OFDM mode.

The data rate in simulation is set to $11 \mathrm{Mbps} \mathrm{CCK}$ modulation with the overlap length set to 8 for simplicity since 8 bits comprise a CCK symbol. The BER versus SNR performance of $11 \mathrm{Mbps}$ under RMS 100ns is shown at Fig. 6. From Fig. 6 we can see that the degradation of implementing overlapping and without overlapping is about $1 \mathrm{~dB}$ and the degradation between perfect channel knowledge and with channel estimation is about $2.5 \mathrm{~dB}$ and $3 \mathrm{~dB}$ to AWGN bound.

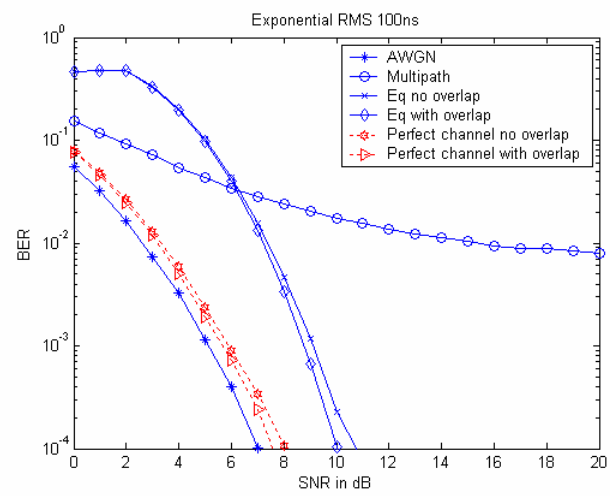

Figure 6. BER versus SNR with RMS delay 100ns

The BER versus SNR performance under the harsh environment of RMS 250ns is shown at Fig. 7. From Fig. 7 we can clearly see that under heavy multipath even with perfect channel knowledge without any overlapping done at frequency domain equalization process the BER will remain high at all SNR.
With channel estimation and overlapping with gives a degradation about 5-6 dB compared with the AWGN bound. A larger overlapping window length can be considered at a larger delay spread environment and the decision of a better choice of the overlapping window length can be made with more extensive analysis with various channel models and other impairments such as carrier offset, timing synchronization and power and computation considerations.

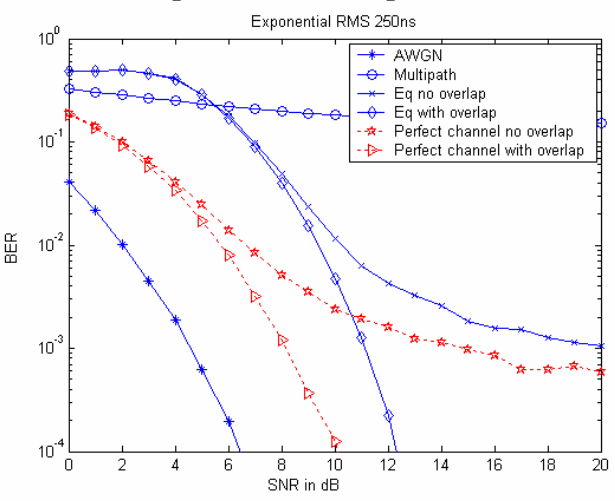

Figure 7. BER versus SNR with RMS delay 250ns

\section{CONCLUSIONS}

In this paper, a frequency domain equalizer for singlecarrier $802.11 \mathrm{~g}$ is proposed. Channel estimation is achieved in frequency domain with modified RLS algorithm. For channel compensation, frequency domain zero-forcing equalization is performed with overlapping sliding windows to reduce the symbol estimation error caused by circularly de-convoluting a linear convolution process without cyclic prefix.

\section{REFERENCE}

[1] IEEE Std 802.11g, June 2003.

[2] S. D. Lingwood, H. Kaufmann, and B. Haller, "ASIC Implementation of a Direct-Sequence Spread-Spectrum RAKE-Receiver," IEEE 44th Vehicular Technology Conference, Stockholm, Sweden, pp.13261330, Jun 8-10 1994.

[3] Li-Mei Chen, Bor-Sen Chen, 'A Robust Adaptive DFE Receiver for DS-CDMA System under Multipath Fading Channels', IEEE transaction, pp. 1523-1532, July 2001.

[4] S. Haykin, Adaptive Filter Theory, 4rd ed., Prentice Hall Inc., Englewood Cliffs, NJ, 2002

[5] D. Mansour and A. H. Gray, "Unconstrained Frequency-Domain Adaptive Filter," IEEE Trans. on Acoustics, Speech, and Signal Processing, vol.30, no.5, Oct. 1982.

[6] G. H. Golub and C. F. van Loan. Matrix Computations. Third Edition, Johns Hopkins, ISBN 0-8018-5413-X, 1996.

[7] Z. Wang and G. B. Giannakis, "Wireless Multicarrier Communications,'IEEE Signal Processing Magazine, vol. 17, no.3, pp. 29-48, May 2000.

[8] M. Vollmer, M. Haardt, J. G"otze, "Comparative Study of JointDetection Techniques for TD-CDMA based Mobile Radio Systems," IEEE Journal on Selected Areas in Communications, vol. 19, no. 8, pp 1461-1475, August 2001.

[9] Karen Halford, Mark Webster, "Multipath Measurements in Wireles LANS “,Application Note AN9895.1, Intersil, 2001. 